\title{
Comparative analyses of Campylobacter concisus strains reveal the genome of the reference strain BAA-1457 is not representative of the species
}

Nadeem O Kaakoush', Nandan P Deshpande ${ }^{2}$, Marc R Wilkins ${ }^{1,2,3}$, Mark J Raftery ${ }^{4}$, Karolina Janitz ${ }^{3}$ and Hazel Mitchell ${ }^{1 *}$

\begin{abstract}
Background: Several studies have shown that significant genotypic heterogeneity exists among Campylobacter concisus strains. Recently, the genome of C. concisus UNSWCD, isolated from a patient with Crohn's disease, was sequenced.

Results: In this study, comparative analyses were performed between strain UNSWCD and BAA-1457, isolated from a patient with acute gastroenteritis. Searches between C. concisus UNSWCD and BAA-1457 showed that 76\% of genes were homologues, whereas those between C. jejuni strains showed 90-91\% to be homologues, indicating substantial variation exists within these two C. concisus genomes. More specific bidirectional homology searches identified 1593 genes that are shared between these strains, and 115 and 281 genes unique to UNSWCD and BAA1457, respectively. Significantly, differences in the type of flagellin glycosylation pathways between the two strains were identified and confirmed by PCR. The protein profiles of UNSWCD, BAA-1457 and a further six strains of C. concisus were compared and analyzed bioinformatically, and this differentiated the strains into four clades. BAA1457 was found to be highly divergent (average similarity: 56.8\%) from the other seven strains (mean average similarity \pm standard deviation: $64.7 \pm 1.7 \%$ ). Furthermore, searches for homologues of the 1593 proteins found to be common between UNSWCD and BAA-1457 were conducted against all available bacterial genomes, and 18 proteins were found to be unique to $C$. concisus, of which 6 were predicted to be secreted, and may represent good markers for detection of this species.
\end{abstract}

Conclusions: This study has elucidated several features that may be responsible for the heterogeneity that exists among C. concisus strains, and has determined that the strain BAA-1457 is genetically atypical to other $C$. concisus strains and is not a good candidate reference strain.

Keywords: Campylobacter concisus, comparative, glycosylation, pseudoaminic acid, legionaminic acid

\section{Background}

Campylobacter concisus is a motile Gram-negative, spiral/curved, bacterium that requires a microaerobic hydrogen-enriched environment for growth [1]. Due to its association with acute enteritis and Crohn's disease (CD) [2-11], C. concisus has been described as an

\footnotetext{
* Correspondence: H.Mitchell@unsw.edu.au

'School of Biotechnology and Biomolecular Sciences, The University of New South Wales, Sydney, NSW 2052, Australia

Full list of author information is available at the end of the article
}

emergent pathogen of the human intestinal tract. However, the isolation of $C$. concisus from healthy individuals, and the failure of some studies to show a significant difference in the prevalence of $C$. concisus in subjects with diarrhea and healthy controls $[4,5]$, has resulted in controversy regarding the role of $C$. concisus in intestinal disease.

Given that $C$. concisus has been reported to be genetically and taxonomically diverse with up to four or more genomospecies being described $[3,12,13]$, we recently

\section{() Biomed Central}


investigated the ability of a range of $C$. concisus strains to attach to and invade human intestinal epithelial cell lines using scanning electron microscopy (SEM) [14]. In that study, the adherence and invasive abilities of $C$. concisus UNSWCD, isolated from a CD patient, were compared with that of $C$. concisus strains UNSWCS and ATCC 51562 isolated from patients with acute gastroenteritis, and ATCC 51561 isolated from a healthy control. Based on the SEM results, C. concisus UNSWCD attached to and appeared to invade host cells [14]. While $C$. concisus strains from acute gastroenteritis or a healthy control also displayed flagellum-mediated attachment via microvilli, these strains did not appear to invade [14]. Based on these findings, Man et al quantified the invasive ability of the four $C$. concisus strains using gentamicin protection assays, and showed that the percentage invasion of $C$. concisus UNSWCD was more than 46- and 200-fold higher than that of C. concisus UNSWCS and C. concisus ATCC 51562, respectively [14]. Interestingly, C. concisus ATCC 51561 isolated from a healthy subject, showed no evidence of invasion.

Man et al further investigated the invasion process of C. concisus UNSWCD and found that host microtubules and microfilaments were involved due to the attenuation of invasion by inhibitors such as colchicine and cytochlasin D [14]. Interestingly, there is some consensus that Campylobacter jejuni strains may also require both microtubules and microfilaments during the invasion process into different intestinal cell lines [15-17], a finding that would suggest that $C$. concisus UNSWCD may use a similar mechanism of invasion to $C$. jejuni. Moreover, Man et al showed that C. concisus UNSWCD preferentially attached to intercellular junctional spaces and that this spatial distribution was concomitantly associated with a loss of membrane-associated $\mathrm{ZO}-1$ and occludin [14].

As a result, Man et al postulated that the differences observed in the pathogenic potential of the $C$. concisus strains may be related to the genetic diversity among them [14]. To investigate this hypothesis, we recently sequenced the genome of $C$. concisus UNSWCD in order to compare it with the only other available C. concisus genome BAA-1457 (also known as strain 13826), a strain isolated from the feces of a patient with acute gastroenteritis [18]. A draft genome was assembled employing the de novo assemblers Velvet and Edena, and the UNSWCD genome size was found to be smaller (1.84 MB) when compared to its reference BAA-1457 counterpart (2.1 MB). Sequence comparisons to identify orthologues, essential gene verification analysis, syntenic association maps and proteomic validations by Orbitrap tandem mass spectrometry (more than $70 \%$ of the proteome identified), revealed a highly accurate assembly but one with significant differences to that of $C$. concisus
BAA-1457. For example, clusters of syntenically placed genes in the $C$. concisus BAA-1457 genome were shown to be absent in the $C$. concisus UNSWCD genome, and thus, the functions associated with these genes are likely to be absent in UNSWCD [18].

In the current study, we analyzed the genomic and proteomic differences between $C$. concisus UNSWCD and BAA-1457 in order to examine the heterogeneity within this species and to identify putative factors responsible for the increased pathogenesis of $C$. concisus UNSWCD. Differences identified in the flagellin glycosylation of $C$. concisus UNSWCD and BAA-1457 were then assessed in a further six $C$. concisus strains.

\section{Results and Discussion}

\section{Degree of diversity within Campylobacter concisus}

Phylogenetic analyses of the 16S rRNA gene, internal transcribed region sequence and 23S rRNA gene of $C$. concisus UNSWCD and BAA-1457 have shown that these bacteria cluster together when compared to other Campylobacter species [19]. However, there remains high genetic diversity between the different $C$. concisus strains $[3,12,13]$. Studies have found that this species comprises several molecular groups (genomospecies). For example, Vandamme et al reported that fecal $C$. concisus isolates exhibited only 42 to $50 \%$ DNA-DNA hybridization values with strains of oral origin [13]. Further studies using pulsed field gel electrophoresis [12] and protein profiling [3] found C. concisus to comprise at least two genomospecies, which were phenotypically indistinguishable, but genetically divergent [12] More recently, Aabenhus et al analyzed the genotype of 62 C. concisus clinical isolates using amplified length fragment polymorphism and showed that $C$. concisus contained at least four distinct genomospecies [2].

The recent sequencing of a second $C$. concisus genome (strain UNSWCD) [18], along with the available BAA-1457 genome provided us with the opportunity to perform comparative analyses of these genomes in an effort to identify differences among $C$. concisus strains. To understand the extent of variability between the genomes of the two C. concisus strains, bidirectional homology searches were performed and the values compared with that of searches between two pairs of the three $C$. jejuni strains with sequenced and comprehensively validated genomes. When cutoff values of $70 \%$ identity plus at least $85 \%$ gene length coverage were employed, searches between $C$. concisus UNSWCD and BAA-1457 showed that $76 \%$ of proteins were homologues, whereas those for C. jejuni NCTC 11168 and 81116 and NCTC 11168 and $81-176$ showed $91 \%$ and $90 \%$ to be homologues, respectively. Calculation of the average percentage identities for all homologues revealed that for C. concisus UNSWCD and BAA-1457 it was $96 \%$ whereas that 
for the two C. jejuni pairs was 98\%. Overall these values clearly indicate that even when compared to a phylogenetically related species, higher variation existed within the $C$. concisus genomes.

Further analyses of the differences in gene content across the genomes of C. concisus BAA-1457 and UNSWCD and other Campylobacter species were performed employing the CGView webserver (Figure 1). As expected, the genome with the highest homology to BAA-1457 was that of UNSWCD. Of interest were eight regions that were present in BAA-1457 yet were absent in all the other Campylobacter species including C. concisus UNSWCD (Figure 1). This provided initial evidence that the higher variation observed between the two $C$. concisus strains may be due to uncommon elements within the reference BAA-1457 strain.

More specific bidirectional homology searches for genes with greater than $40 \%$ similarity between the genomes of UNSWCD and BAA-1457 were performed. In addition, genes with $30-40 \%$ similarity were manually verified using functional domain and gene ontology analyses. This identified 1593 genes that are shared between these strains, representing a genome percentage similarity of $91.7 \%(1593 / 1738)$ for UNSWCD and $79.2 \%$ (1593/2010) for BAA-1457. The searches also identified a total of $115(6.6 \%)$ and $281(14.0 \%)$ genes unique to UNSWCD and BAA-1457, respectively (Additional file 1, Table S1; Additional file 2, Table S2), with a further 30 and 136 genes that were not categorized into either group due to insufficient information on these genes.

\section{Genomic variations unique to Campylobacter concisus BAA-1457}

Of the 281 genes unique to BAA-1457 when compared to UNSWCD, 111 proteins they encoded were at a level of abundance within the cell that could be detected by mass spectrometric analyses [18]. The finding that these proteins were expressed when the bacterium was grown under normal conditions would indicate that they play an important role within the cell. The 281 genes encoded proteins with a variety of functions, including transport systems, energy metabolism and a secretion system, as well as 130 hypothetical proteins. Of specific interest was the identification of at least four proteins involved in a Type VI secretion apparatus, CCC13826_1177, CCC13826_1178, CCC13826_1182 and CCC13826_1188, all of which were expressed under normal conditions (Additional file 1, Table S1). Type VI secretion systems (T6SS) have been identified in many bacterial pathogens including Vibrio cholerae, Pseudomonas aeruginosa, Yersinia pestis and Salmonella enterica, and have been implicated in the virulence of all of these species [20]. Although T6SS are employed to transport proteins across the bacterial envelope, more recently, a family of T6SS proteins have been found to share structural features with the cell-puncturing device of the T4 bacteriophage, and thus, may be used by the bacterium to puncture host cell membranes and insert the T6SS apparatus into the host cytosol [21].

Another protein of interest found within the unique proteins of BAA-1457 was the zonula occludens toxin (Zot). This protein and its possible functions within the cell have been previously discussed by Kaakoush et al [22]. Briefly, Zot is known to mimic a physiological modulator of intercellular tight junctions [23], and is used by virulent pathogens such as Vibrio cholerae and Neisseria meningitidis to increase tissue permeability [24]. The presence of Zot in BAA-1457 and its absence in UNSWCD was confirmed by PCR (Figure 2A). The presence of this gene in a further six strains of $C$. concisus (Table 1) was also investigated, and the toxin was found to be absent in all six strains (Figure 2A) (results were confirmed with the second primer pair ZotF/Zot2), suggesting that it is an uncommon feature within the genomes of $C$. concisus strains.

\section{Genomic variations unique to Campylobacter concisusUNSWCD}

Due to the isolation of UNSWCD from a patient with $\mathrm{CD}$ and its increased invasive potential as compared with other $C$. concisus strains [14], the unique features within this strain compared to BAA-1457 were of particular interest. The 115 genes unique to UNSWCD encoded 37 proteins that were at a level of abundance within the cell that could be detected by mass spectrometric analyses (Additional file 2, Table S2) [18]. They comprised proteins with functions including transport, oxidative stress and carbohydrate metabolism, as well as 88 hypothetical proteins. For example, a heat shock protein $\mathrm{G}$ homologue found to be involved in protection against oxidative stress in several bacterial species including Cyanobacteria was identified [25].

Several proteins involved in phage protection were also identified, including a phage portal protein, and a phage antirepressor protein. In addition, a modification methylase and endonuclease were identified. Modification methylases are involved in restriction-modification systems that are responsible for producing a speciescharacteristic methylation pattern in a short sequence in the host cell's DNA. Thus, any DNA from another species that gains entry into the cell and lacks the characteristic methylation pattern are recognized by restriction endonucleases and destroyed by cleavage. In addition, proteins involved in the processing of exogenous DNA and DNA repair were found, namely a competence protein CoiA and a nucleotidyltransferase family protein. While there are limited studies on the function of CoiA, Desai and Morrison have reported that this protein 


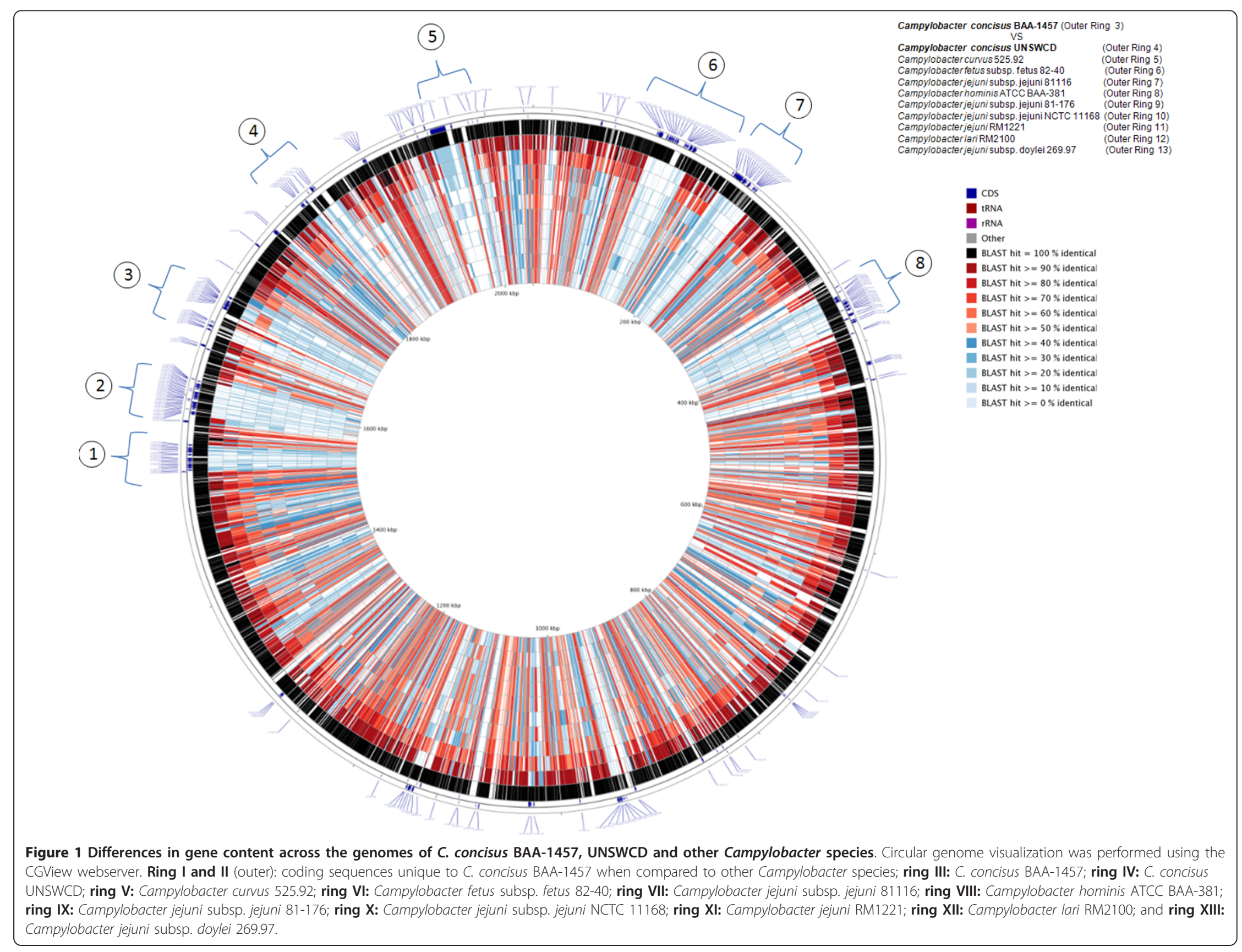




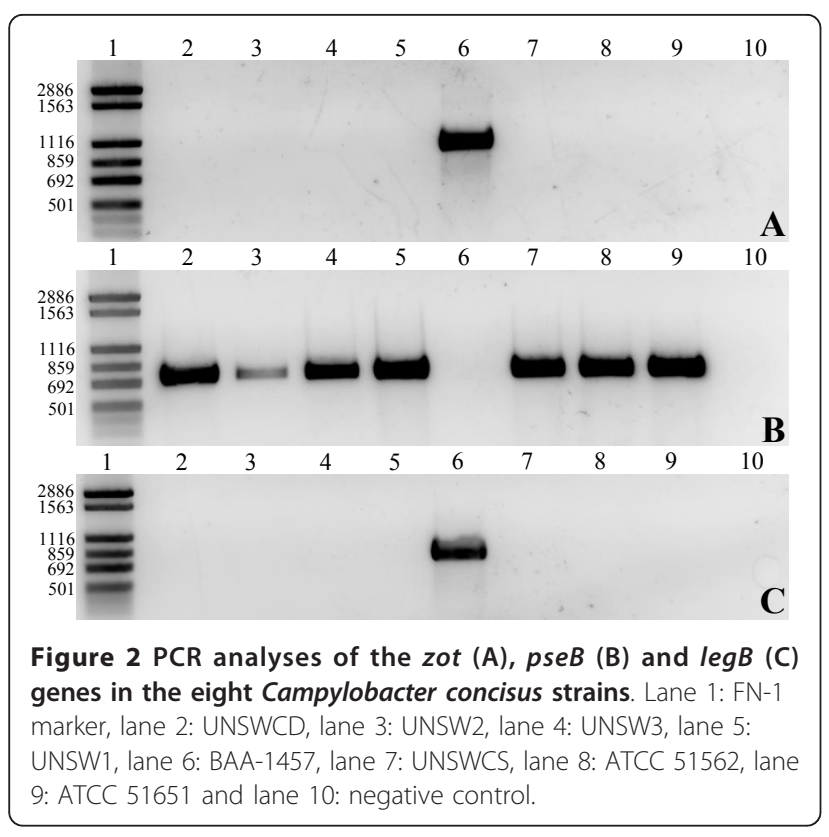

although not involved in uptake of donor DNA, is involved in processing this DNA to make viable mutants [26]. The presence of these proteins in UNSWCD may suggest that this strain has a more adaptable nature that may have lead to its increased efficiency in the host.

A further protein, an $\mathrm{O}$-antigen ligase, that may play a role in the aggregation and adherence of $C$. concisus UNSWCD onto host cells, was also identified. This protein functions in the attachment of polymerized O-antigen repeat units to the lipid A core, and has been found to be important for cell wall integrity and bacterial motility [27]. More recently, Morgenstein et al found that deactivation of the $\mathrm{O}$-antigen ligase in Proteus mirabilis had little effect on its swimming motility in soft agar but blocked its swarming motility on solid surfaces [27]. Thus, this protein may aid C. concisus UNSWCD to aggregate on host cells as has been observed previously by SEM [14].

Another protein of interest that was unique to C. concisus UNSWCD when compared to BAA-1457, and to other members of the Campylobacterales, was the Acr protein ( $\alpha$-crystallin). Interestingly, expanded searches revealed that the gene encoding this protein is found in the genomes of extreme $\varepsilon$-Proteobacteria such as Nautilia profundicola and Caminibacter mediatlanticus. In addition, Mycobacterium tuberculosis has been shown to have two members of the Acr family of molecular chaperones. For example, in 1998, Yuan et al demonstrated that acr transcription and Acr expression were strongly induced by hypoxic conditions and in vitro infection of macrophages, respectively [28]. These authors hypothesized that this protein was important for long term viability and replication during initial M. tuberculosis infections. More recently, Wilkinson et al determined that two Acr proteins, Acr1 and Acr2, were present in $M$. tuberculosis and were important for the bacterium's interaction with the host [29]. Acr1 has been shown to be induced by exposure to hypoxia or nitric oxide and is associated with bacterial persistence in a non-replicating state, while Acr2 is induced by heat shock, oxidative stress, and uptake by macrophages. In 2005, Stewart et al demonstrated that both Acr proteins contribute to persistent infection with $M$. tuberculosis, and suggested that manipulation of acr expression can influence the host response to infection [30]. More recently, Stewart et al showed that deletion of the acr gene resulted in infection of C57BL6 mice with bacillary loads 1-2 log units higher than the wild-type strain [31]. In addition, mice infected with the mutant strain showed high levels of TNF- $\alpha$, IFN- $\gamma$ and G-CSF in their serum, suggesting that Acr may play a role in modulating the host response to infection [31]. The presence of this protein in UNSWCD and not in BAA-1457 or any other Campylobacterales further supports the invasive nature of this $C$. concisus strain within the host.

\section{Flagellin glycosylation in Campylobacter concisus}

Like many Gram-negative bacteria with polar flagella, Campylobacter flagellins are known to undergo glycosylation. Although pathway variations and sugar modifications exist among strains, most $C$. jejuni strains contain genes for the synthesis of two sugars that link to flagellin: pseudaminic acid (PA) and an acetamidino form of

Table 1 Campylobacter concisus strains used in this study.

\begin{tabular}{llll}
\hline Strain & Source of isolation & Disease & Reference \\
\hline UNSWCD & Human intestinal biopsy & Crohn's disease & {$[11]$} \\
UNSW1 & Human intestinal biopsy & Chronic gastroenteritis & [Kaakoush et al, unpublished data] \\
UNSW2 & Human intestinal biopsy & Crohn's disease & [Kaakoush et al, unpublished data] \\
UNSW3 & Human intestinal biopsy & Crohn's disease & [Kaakoush et al, unpublished data] \\
ATCC 51561 & Human feces & Healthy & {$[13]$} \\
ATCC 51562 & Human feces & Acute gastroenteritis & {$[13]$} \\
UNSWCS & Human feces & Acute gastroenteritis & {$[14]$} \\
BAA-1457 & Human feces & Acute gastroenteritis & {$[41]$} \\
\hline
\end{tabular}


legionaminic acid (LA) [32-34]. The entire PA biosynthetic pathway has been characterized in Helicobacter pylori and has been shown to involve six enzymes PseB, PseC, PseH, PseG, PseI and PseF [35]. More recently, the LA biosynthetic pathway was characterized in $C$. jejuni and has also been shown to involve six enzymes, namely LegB, LegC, LegH, LegG, LegI and LegF [36].

Bidirectional homology searches against the genomes of UNSWCD and BAA-1457 revealed that UNSWCD contained the PA biosynthetic pathway whereas BAA1457 contained the LA biosynthetic pathway. To further confirm these findings, a phylogenetic analysis was performed on the six proteins of each pathway for several Campylobacterales (Figure 3). There was a distinct division between the two types of pathways, with the PA and LA pathways clustering into separate groups. Importantly, the six proteins from UNSWCD clustered with the PA pathways whereas the six proteins from BAA-1457 clustered with the LA pathways, confirming the homology searches. C. concisus BAA1457 was the only member of the Campylobacterales to express only a LA biosynthetic pathway. Interestingly, the PA biosynthetic pathways of Campylobacter

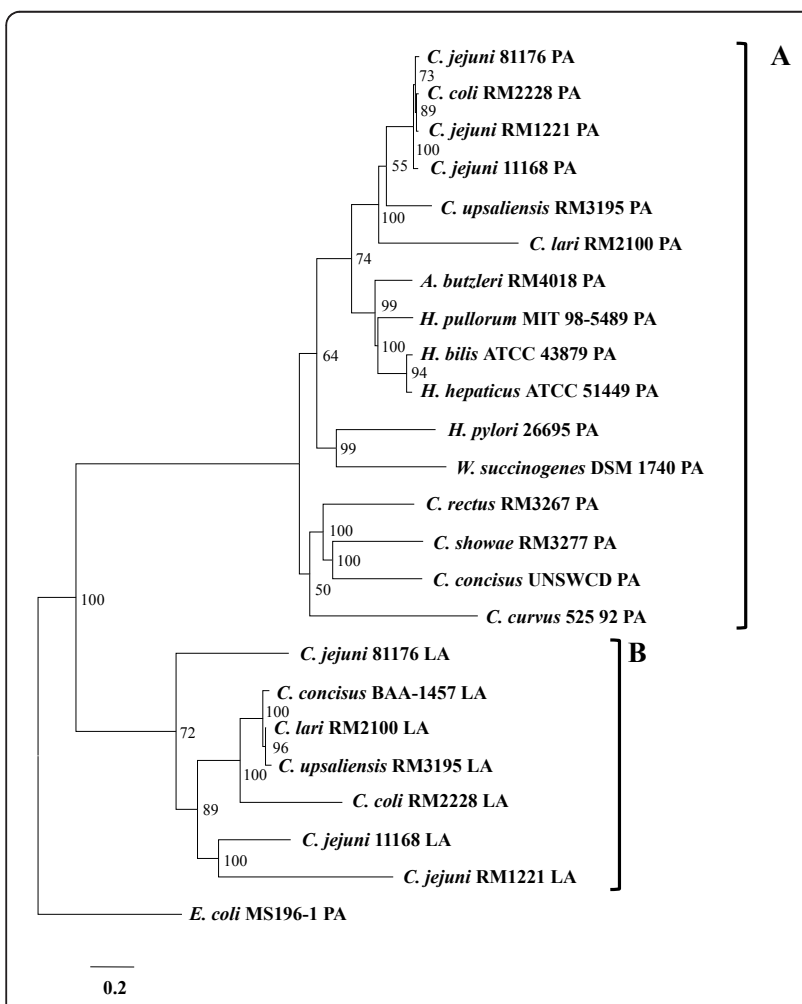

Figure 3 Phylogenetic analysis of the pseudaminic acid and legionaminic acid biosynthetic pathways of Campylobacterales. Trees were constructed at the Mobyle@Pasteur portal using the default settings. Trees were visualized using the TreeView software. $0.2=$ base substitutions per site. species that contained both pathways within their genome clustered together, revealing an evolutionary closeness within the flagellin glycosylation pathways of these bacteria.

The presence of these pathways were further verified experimentally, where the presence of $p s e B$ in UNSWCD (Figure 2B) and $\operatorname{leg} B$ in BAA-1457 (Figure 2C) was confirmed by PCR. Six additional C. concisus strains (Table 1 ) were also analyzed, and it was found that they all contained the PA biosynthetic pathway (Figure 2B, C). While currently the function of flagellin glycosylation in Campylobacter spp. is not fully understood, perturbations in flagellin glycosylation systems of Campylobacter spp. and $H$. pylori have been shown to result in negative effects on filament assembly, autoagglutination, microcolony formation on intestinal epithelial cells, cyto-adherence and invasion [32]. Thus, it is possible that differences in the flagellin glycosylation pathways of $C$. concisus strains may be involved in the variations seen in their adherence and invasive potential. In addition, this provided further evidence that $C$. concisus BAA-1457 was genetically divergent from the other C. concisus strains studied. Their similar genetic backgrounds and the presence of only a PA biosynthetic pathway in the seven $C$. concisus strains and only a LA biosynthetic pathway in BAA-1457 makes these bacteria ideal organisms to study these flagellin glycosylation pathways in more detail.

\section{Protein profiles of Campylobacter concisus}

The genomic variations between UNSWCD and BAA1457 led us to investigate the protein profiles of these two strains and a further six C. concisus strains (Table 1) isolated from different disease states. The protein profiles of the total cell lysates of the eight strains were determined using SDS-PAGE, and analyzed using the Phoretix 1D pro software, which clustered the strains based on their banding patterns (Figure 4A, B). The eight strains did not cluster according to the disease state from which they were isolated. This is in line with the findings of Engberg et al who reported that strainspecific differences in the ability of $C$. concisus to induce cytolethal distending toxin-like effects on monkey kidney cells had no specific association with disease outcome [4]. Moreover, disease state was not a factor in the hemolytic phospholipase $\mathrm{A}_{2}$ activity of $C$. concisus strains isolated from children with diarrhea [37].

At a distance of 0.4 two clades were present, one containing BAA-1457 and the other all other seven strains (Figure 4B). The average similarity of BAA-1457 with the other seven strains was $56.8 \%$, while the mean average similarity of the other seven strains was $64.7 \%$ with a standard deviation of $1.7 \%$ (Table 2). This indicated that BAA-1457 is highly divergent from the other $C$. 


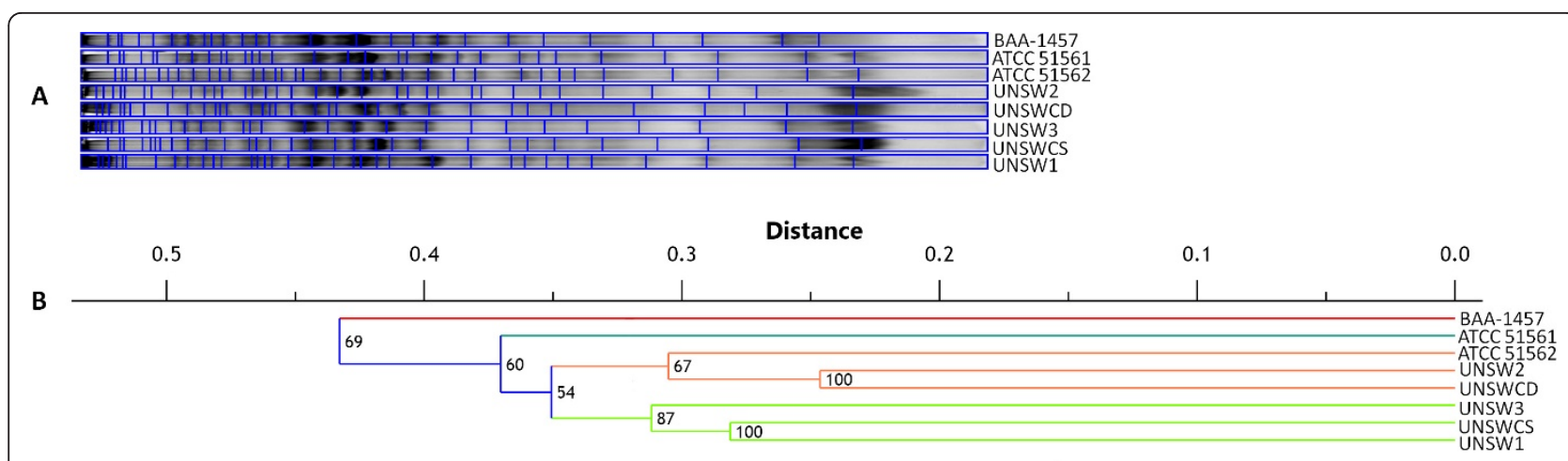

Figure 4 Protein profiles of eight Campylobacter concisus strains. (A) Phoretix 1D pro was employed to cluster the lanes based on banding patterns. (B) Dendrogram is a Unweighted Pair Group Method with Arithmetic Mean (UPGMA) Dice coefficient distance tree.

concisus strains, providing additional evidence that it is atypical in nature. Interestingly, at a distance of 0.35 , three clades were present, one containing BAA-1457, one containing ATCC 51561 isolated from a healthy control and the third containing the other six strains. Excluding the results of BAA-1457, the average similarity of ATCC 51561 with the six other strains was $63.2 \%$, while the average similarity of the six other strains ranged from 64.7-69.0\% (mean average similarity of the six other strains $=66.5 \pm 1.7 \%$ ) (Table 2), indicating that the healthy control strain was divergent from the other strains isolated from acute and chronic gastroenteritis and CD. Finally, at a distance of 0.325 four clades were present, where the previous third cluster comprising six strains was split into two groups of three strains (group 1: UNSWCD, UNSW2 and ATCC 51562; group 2: UNSW3, UNSW1 and UNSWCS). While this division was not based on disease state, it may shed light on the discrepancies in the results of previous studies differentiating C. concisus strains. That is, techniques with the capacity of differentiating up to two $C$. concisus genomospecies may be observing what is seen at a distance of 0.4, whereas those differentiating up to four molecular groups may be observing that seen at a distance of 0.325 .

\section{Possible markers for the identification of Campylobacter concisus}

Due to the large variability observed between $C$. concisus strains UNSWCD and BAA-1457, proteins found in both strains but not in another bacterial species may be ideal markers for the detection of this species. Searches for homologues of the 1593 proteins found to be common between the two strains were conducted against all available bacterial genomes, and 18 proteins were found to be unique to C. concisus, of which six were bioinformatically predicted to be secreted (Table 3 ). These were made up of mostly short ( 40-50 amino acids) hypothetical proteins. Of interest was CCC13826_2290 that was 160 amino acids in length and predicted to contain a signal peptide (Table 3). Given that secreted or membrane proteins are more likely to illicit a host immune response, these identified proteins make good targets for species-specific detection through methods such as

Table 2 Percentage average similarities of the protein profiles of eight Campylobacter concisus strains as calculated by Phoretix 1D pro.

\begin{tabular}{|c|c|c|c|c|c|c|c|c|}
\hline \multirow[b]{2}{*}{ Strain } & \multicolumn{8}{|c|}{ Average similarity (\%) } \\
\hline & UNSWCD & UNSW2 & UNSW3 & UNSW1 & BAA-1457 & UNSWCS & ATCC 51562 & ATCC 51561 \\
\hline UNSWCD & 100 & 75 & 62 & 72 & 49 & 71 & 73 & 61 \\
\hline UNSW2 & 75 & 100 & 73 & 62 & 57 & 59 & 66 & 68 \\
\hline UNSW3 & 62 & 73 & 100 & 70 & 62 & 68 & 55 & 60 \\
\hline UNSW1 & 72 & 62 & 70 & 100 & 57 & 72 & 69 & 62 \\
\hline BAA-1457 & 49 & 57 & 62 & 57 & 100 & 68 & 45 & 60 \\
\hline UNSWCS & 71 & 59 & 68 & 72 & 68 & 100 & 61 & 62 \\
\hline ATCC 51562 & 73 & 66 & 55 & 69 & 45 & 61 & 100 & 66 \\
\hline ATCC 51561 & 61 & 68 & 60 & 62 & 60 & 62 & 66 & 100 \\
\hline Mean $\pm S D^{*}$ & $66.1 \pm 9.3$ & $65.7 \pm 6.8$ & $64.3 \pm 6.3$ & $66.3 \pm 5.9$ & $56.8 \pm 7.8$ & $65.9 \pm 5.1$ & $62.1 \pm 9.5$ & $62.7 \pm 3.1$ \\
\hline
\end{tabular}

Error on the mean is the standard deviation (SD).

*Mean of percentage similarities \pm standard deviation excludes the $100 \%$ value 
Table 3 Proteins specific to Campylobacter concisus.

\begin{tabular}{|c|c|c|c|}
\hline BAA-1457 ORF & Protein name & Protein length (aa) & Secreted \\
\hline$\overline{C C C 13826 \_0031}$ & Hypothetical protein & 64 & No \\
\hline CCC13826_0210 & Hypothetical protein & 56 & No \\
\hline CCC13826_0311 & Hypothetical protein & 42 & Yes $\left(\operatorname{Sec}^{*}=0.61\right.$ \\
\hline CCC13826_0334 & Aspartokinase & 52 & Yes $\left(\operatorname{Sec}^{*}=0.57\right.$ \\
\hline CCC13826_0382 & Outer membrane protein & 44 & No \\
\hline CCC13826_0423 & Transmembrane transport protein & 41 & Yes $\left(\right.$ SigP $\left.P^{\#}=0.98\right)$ \\
\hline CCC13826_0586 & Signal transduction sensor protein & 38 & No \\
\hline CCC13826_0742 & Hypothetical protein & 40 & No \\
\hline CCC13826_0909 & Hypothetical protein & 40 & Yes $\left(\operatorname{Sec}^{*}=0.92\right.$ \\
\hline CCC13826_1038 & Hypothetical protein & 41 & No \\
\hline CCC13826_1698 & L-cystine binding protein TcyA & 179 & No \\
\hline CCC13826_1719 & Hypothetical protein & 52 & No \\
\hline CCC13826_1918 & Hypothetical protein & 44 & Yes $\left(\operatorname{Sec} P^{*}=0.70\right.$ \\
\hline CCC13826_2161 & Ferric uptake regulation protein & 47 & No \\
\hline CCC13826_2242 & Periplasmic protein & 41 & No \\
\hline CCC13826_2291 & Hypothetical protein & 38 & No \\
\hline CCC13826_2290 & Hypothetical protein & 160 & Yes $\left(\right.$ SigP $\left.P^{\#}=1.0\right)$ \\
\hline CCC13826_2294 & Hypothetical protein & 45 & No \\
\hline
\end{tabular}

Proteins common between C. concisus UNSWCD and BAA-1457 with no homologues in other bacterial species were identified through blastp searches. Proteins were predicted to be secreted through the SignalP and SecretomeP servers.

* SecP $=$ Secretome $P$ prediction

\# SigP $=$ SignalP prediction

ELISA. In addition, the low number of proteins unique to these two strains further emphasizes the extent of variability between them.

Validation of the presence of the six genes encoding putative taxon-specific markers bioinformatically predicted to be secreted (Table 3 ) in the eight $C$. concisus strains was performed using PCR and sequencing. Two of the six genes (ccc13826_0334 and ccc13826_2290) were present within all the eight $C$. concisus strains, thus, providing evidence that they may be good taxonspecific markers for this bacterium. Further investigation, within more $C$. concisus strains, of the potential of these genes to be taxon-specific markers is required.

\section{Conclusions}

This study has elucidated several features that may be responsible for the heterogeneity that exists among $C$. concisus strains, and has identified factors that may explain the differences in their pathogenic potential. The identification of different virulence factors ( $\alpha$-crystallin and zonula occludens toxin) within the two $C$. concisus strains (UNSWCD and BAA-1457) as well as the different types of flagellin glycosylation biosynthetic pathways (pseudaminic acid and legionaminic acid) present interesting avenues to further investigate the differences in pathogenesis of $C$. concisus strains. Importantly, based on the current study, it would appear that BAA-1457 is atypical to other C. concisus strains, and thus, as a reference strain for sequence- based analyses on this species, UNSWCD may represent a better candidate.

\section{Methods}

\section{Comparative bioinformatic analyses}

Homology searches were performed using the blastp and blastn tools through the National Center for Biotechnology Information (NCBI) website available at (http:// www.ncbi.nlm.nih.gov/) using the default settings. Comparative bioinformatic analyses on the genomes of $C$. concisus strains UNSWCD and BAA-1457 were performed using the Blast2GO tool [38] available at (http:// www.blast2go.org/start_blast2go). The Kyoto Encyclopedia of Genes and Genomes (KEGG) [39] available at (http://www.genome.jp/kegg) was employed to determine the biochemical pathways to which genes were assigned. Circular genome visualization was performed using the CGView web-server [40]. Phylogenetic trees on the glycosylation pathways within Campylobacterales were performed at the Mobyle@Pasteur portal available at (http://mobyle.pasteur.fr/cgi-bin/portal.py\#welcome) using the default settings. Trees were visualized using the TreeView software available at (http://taxonomy. zoology.gla.ac.uk/rod/treeview.html). The presence and location of signal peptide cleavage sites in the amino acid sequences were predicted using the default settings for Gram-negative bacteria on the SignalP Server 3.0 (http://www.cbs.dtu.dk/services/SignalP/). Non-classically secreted proteins were predicted using the 
SecretomeP 2.0 Server (http://www.cbs.dtu.dk/services/ SecretomeP/).

\section{Bacterial cultures}

Campylobacter concisus strains UNSWCD [11], UNSW1, UNSW2, UNSW3 [Kaakoush et al, unpublished data], ATCC 51561 [13], ATCC 51562 [13], UNSWCS [14] and BAA-1457 [41] (Table 1) were grown on Horse Blood agar (HBA) supplemented with $6 \%$ defibrinated horse blood. Cultures were incubated at $37^{\circ} \mathrm{C}$ under microaerobic conditions generated using Campylobacter Gas Generating Kits BR0056A (Oxoid). The purity of bacterial cultures was confirmed by motility and morphology observed under phase contrast microscopy. Strains were selected based on the diagnosis of the patient they were isolated from because of the observed differences in the pathogenic potential of strains isolated from chronic intestinal diseases and acute intestinal diseases ([14], Kaakoush et al, unpublished data).

\section{Extraction of genomic DNA, polymerase chain reactions and sequencing}

DNA extraction was performed using the Puregene Core Kit A (Qiagen) according to the manufacturer's instructions. The concentration and quality of DNA was measured using a Nanodrop ND-1000 Spectophotometer (Nanodrop Technologies). PCR reactions were performed on the DNA extracted from the $C$. concisus strains. The Zot PCRs were performed using the primer pair Zot1 (GCAACTTAGAAAAAGTATCGG) and Zot2 (TAATAGTTCTCGATGAAGCC), which amplify a 979 bp region, and the primer pair ZotF (CTAGAATCAGTTTGTGGAGAT) and Zot2, which amplify a 790 bp region. The pseB PCR was performed using the primer pair pseB-F (CTAGTTATCTACTCACGCGAC) and $p s e B-\mathrm{R}$ (GGATAGACGGACTAATAACG), which amplifies a 754 bp region. The legB PCR was performed using the primer pair legB-F (TAGTTATAGGTGCAGCAGGCT) and $\operatorname{leg} B-\mathrm{R}$ (GCATCATCTGCACTATATGGT), which amplifies a 817 bp region. The thermal cycling conditions for all three reactions were: $94^{\circ} \mathrm{C}$ for $5 \mathrm{~min}, 30$ cycles of $94^{\circ} \mathrm{C}$ for $20 \mathrm{~s}, 53^{\circ} \mathrm{C}$ for $20 \mathrm{~s}$, and $72^{\circ} \mathrm{C}$ for $1 \mathrm{~min}$, followed by $72^{\circ} \mathrm{C}$ for $5 \mathrm{~min}$.

To validate possible taxon-specific markers six primer pairs targeting the genes ccc13826_0311, ccc13826_0334, ccc13826_0423, ccc13826_0909, ccc13826_1918 and ccc13826_2290 were designed. The ccc13826_0311 PCR was performed using the primer pair 0311F (GAGGTTAGAAATAACAACAGC) and 0311R (TTGCTTGT TCTCATCGTCGTA), which amplify a 226 bp region. The ccc13826_0334 PCR was performed using the primer pair 0334F (GAGTGATGATTTTGACTACG) and 0334R (CGAGTCGTAACTATCCTCATC), which amplify a 151 bp region. The ccc13826_0423 PCR was performed using the primer pair 0423F (GAAGCATCTAAGCCTGACAGA) and 0423R (ACTCTGCAAACTGACACCAAG), which amplify a 240 bp region. The ccc13826_0909 PCR was performed using the primer pair 0909F (GTTAACATAGCTTTGGCAAGG) and 0909R (GCTGTTTTGCGT AAATTTGTG), which amplify a 98 bp region. The ccc13826_1918 PCR was performed using the primer pair 1918F (GTGCTTAACAAAAGATGTGGC) and 1918R (GCTGTTTTGCG TAAATTTGTG), which amplify a 114 bp region. The ccc13826_2290 PCR was performed using the primer pair 2290F (GAGAGTTTTAGATTTGATGG) and 2290R (CTCATACGAAAGCATCAAGAC), which amplify a $309 \mathrm{bp}$ region. The thermal cycling conditions for all six reactions were: $94^{\circ} \mathrm{C}$ for $5 \mathrm{~min}, 30$ cycles of $94^{\circ} \mathrm{C}$ for $20 \mathrm{~s}, 50^{\circ} \mathrm{C}$ for $20 \mathrm{~s}$, and $72^{\circ} \mathrm{C}$ for $20 \mathrm{~s}$, followed by $72^{\circ} \mathrm{C}$ for $5 \mathrm{~min}$

To confirm the identities of the products, they were first purified using the QIAquick ${ }^{\circledR}$ PCR Purification Kit (Qiagen) according to manufacturer's instructions. Subsequently, sequencing of the positive PCR products was undertaken using the BigDye ${ }^{\mathrm{TM}}$ terminator chemistry (Applied Biosystems). Sequences were resolved on an Automated DNA Sequence Analyser ABI3730 (Applied Biosystems) at the sequencing facility of the School of Biotechnology and Biomolecular Sciences, The University of New South Wales, Sydney, Australia. Products were confirmed using blastn searches available through NCBI.

\section{SDS-PAGE}

Campylobacter concisus strains were grown on HBA plates, and bacteria were washed twice in phosphate buffered saline. Following the final wash, packed cells were resuspended in $1 \mathrm{ml}$ TSU buffer (50 mM Tris $\mathrm{pH} 8.0$, $0.1 \% \mathrm{SDS}, 2.5 \mathrm{M}$ urea) and lysed by two freeze-thaw cycles in liquid nitrogen. Lysate proteins $(20 \mu \mathrm{g})$ were resuspended in 1:1 SDS-PAGE sample buffer $(0.375 \mathrm{M}$ Tris $\mathrm{pH}$ 6.8, 0.01\% SDS, 20\% glycerol, $40 \mathrm{mg} \mathrm{ml}^{-1} \mathrm{SDS}, 31 \mathrm{mg} \mathrm{ml}^{-1}$ DTT, $1 \mu \mathrm{g} \mathrm{ml}^{-1}$ bromophenol blue). For electrophoretic analyses proteins were further denatured by heating at $95^{\circ}$ $\mathrm{C}$ for $5 \mathrm{~min}$. Proteins were separated on 12\% SDS-PAGE gels by electrophoresis for $1.5 \mathrm{~h}$ at $100 \mathrm{~V}$. Gels were stained using Coomassie Brilliant Blue G-250 (Bio-Rad). Phoretix 1D pro software (TotalLab Ltd.; Newcastle Upon Tyne, NE, UK) was employed to determine the percentage similarities between the protein profiles of the C. concisus strains, and to cluster the lanes based on banding patterns.

\section{Additional material}

Additional file 1: Table S1. Strain-specific proteins in Campylobacter concisus BAA-1457. Proteins of $C$. concisus BAA-1457 with no

homologues in UNSWCD were identified through blastp searches. 
Proteins were determined to be expressed through Orbitrap mass spectrometry analyses [18].

Additional file 2: Table S2. Strain-specific proteins in Campylobacter concisus UNSWCD. Proteins of $C$. concisus UNSWCD with no

homologues in BAA-1457 were identified through blastp searches. Proteins were determined to be expressed through Orbitrap mass spectrometry analyses [18].

\section{Acknowledgements}

This work was made possible by the support of the National Health and Medical Research Council, Australia. ND and MRW wish to acknowledge funding support from the EIF Super Science Scheme, the NSW State Government Science Leveraging Fund and The University of New South Wales.

\section{Author details}

${ }^{1}$ School of Biotechnology and Biomolecular Sciences, The University of New South Wales, Sydney, NSW 2052, Australia. ${ }^{2}$ Systems Biology Initiative, School of Biotechnology and Biomolecular Sciences, The University of New South Wales, Sydney, NSW 2052, Australia. ${ }^{3}$ Ramaciotti Centre for Gene Function Analysis, The University of New South Wales, Sydney, NSW 2052, Australia. ${ }^{4}$ Biological Mass Spectrometry Facility, The University of New South Wales, Sydney, NSW 2052, Australia.

\section{Authors' contributions}

NOK participated in the study design, carried out the genetic and proteomic studies, participated in the bioinformatic studies and drafted the manuscript. NPD participated in the study design, carried out the bioinformatic studies and helped to draft the manuscript. MRW participated in the study design and bioinformatic studies and helped to draft the manuscript. MJR participated in the proteomics studies. KJ participated in the genetic studies. HM conceived the study, and participated in its design and helped to draft the manuscript. All authors read and approved the final manuscript.

\section{Competing interests}

The authors declare that they have no competing interests.

Received: 12 September 2011 Accepted: 13 October 2011

Published: 13 October 2011

\section{References}

1. Vandamme P, Dewhirst FE, Paster BJ, On SLW: Campylobacter. In Bergey's Manual of Systematic Bacteriology. Edited by: Brenner DJ, Krieg NR, Staley JT. New York: Springer; 2005:1147-1168

2. Aabenhus R, Permin H, Andersen LP: Characterization and subgrouping of Campylobacter concisus strains using protein profiles, conventional biochemical testing and antibiotic susceptibility. Eur I Gastroenterol Hepatol 2005, 17:1019-1024.

3. Aabenhus R, Permin H, On SL, Andersen LP: Prevalence of Campylobacter concisus in diarrhoea of immunocompromised patients. Scand J Infect Dis 2002, 34:248-252.

4. Engberg J, Bang DD, Aabenhus R, Aarestrup FM, Fussing V, Gerner-Smidt P: Campylobacter concisus: an evaluation of certain phenotypic and genotypic characteristics. Clin Microbiol Infect 2005, 11:288-295.

5. Engberg J, On SL, Harrington CS, Gerner-Smidt P: Prevalence of Campylobacter, Arcobacter, Helicobacter, and Sutterella spp. in human fecal samples as estimated by a reevaluation of isolation methods for Campylobacters. J Clin Microbiol 2000, 38:286-291.

6. Lastovica AJ, le Roux E: Efficient isolation of campylobacteria from stools. J Clin Microbiol 2000, 38:2798-2799.

7. Lindblom GB, Sjogren E, Hansson-Westerberg J, Kaijser B: Campylobacter upsaliensis, C. sputorum sputorum and C. concisus as common causes of diarrhoea in Swedish children. Scand J Infect Dis 1995, 27:187-188.

8. Maher M, Finnegan C, Collins E, Ward B, Carroll C, Cormican M: Evaluation of culture methods and a DNA probe-based PCR assay for detection of Campylobacter species in clinical specimens of feces. J Clin Microbiol 2003, 41:2980-2986.
9. Man SM, Zhang L, Day AS, Leach ST, Lemberg DA, Mitchell H: Campylobacter concisus and other Campylobacter species in children with newly diagnosed Crohn's disease. Inflamm Bowel Dis 2009, 16:1008-1016.

10. Musmanno RA, Russi M, Figura N, Guglielmetti P, Zanchi A, Signori R, Rossolini A: Unusual species of campylobacters isolated in the Siena Tuscany area, Italy. New Microbiol 1998, 21:15-22.

11. Zhang L, Man SM, Day AS, Leach ST, Lemberg DA, Dutt S, Stormon M, Otley A, O'Loughlin EV, Magoffin A, Ng PH, Mitchell H: Detection and isolation of Campylobacter species other than C. jejuni from children with Crohn's disease. J Clin Microbiol 2009, 47:453-455.

12. Matsheka MI, Elisha BG, Lastovica AL, On SL: Genetic heterogeneity of Campylobacter concisus determined by pulsed field gel electrophoresisbased macrorestriction profiling. FEMS Microbiol Lett 2002, 211:17-22.

13. Vandamme P, Falsen E, Pot B, Hoste B, Kersters K, De Ley J: Identification of EF group 22 campylobacters from gastroenteritis cases as Campylobacter concisus. J Clin Microbiol 1989, 27:1775-1781.

14. Man SM, Kaakoush NO, Leach ST, Nahidi L, Lu HK, Norman J, Day AS, Zhang L, Mitchell HM: Host attachment, invasion, and stimulation of proinflammatory cytokines by Campylobacter concisus and other nonCampylobacter jejuni Campylobacter species. J Infect Dis 2010, 202:1855-1865.

15. De Melo MA, Gabbiani G, Pechere JC: Cellular events and intracellular survival of Campylobacter jejuni during infection of HEp-2 cells. Infect Immun 1989, 57:2214-2222.

16. Konkel ME, Joens LA: Adhesion to and invasion of HEp-2 cells by Campylobacter spp. Infect Immun 1989, 57:2984-2990.

17. Oelschlaeger TA, Guerry P, Kopecko DJ: Unusual microtubule-dependent endocytosis mechanisms triggered by Campylobacter jejuni and Citrobacter freundii. Proc Natl Acad Sci USA 1993, 90:6884-6888.

18. Deshpande NP, Kaakoush NO, Mitchell H, Janitz K, Raftery MJ, Li SS, Wilkins MR: Sequencing and Validation of the Genome of a Campylobacter concisus Reveals Intra-Species Diversity. PLoS One 2011, 6: e22170.

19. Man SM, Kaakoush NO, Octavia S, Mitchell H: The internal transcribed spacer region, a new tool for use in species differentiation and delineation of systematic relationships within the Campylobacter genus. Appl Environ Microbiol 2010, 76:3071-3081.

20. Pukatzki S, Ma AT, Revel AT, Sturtevant D, Mekalanos JJ: Type VI secretion system translocates a phage tail spike-like protein into target cells where it cross-links actin. Proc Natl Acad Sci USA 2007, 104:15508-15513.

21. Pukatzki S, MCAuley SB, Miyata ST: The type VI secretion system: translocation of effectors and effector-domains. Curr Opin Microbiol 2009, 12:11-17.

22. Kaakoush NO, Man SM, Lamb S, Raftery MJ, Wilkins MR, Kovach Z, Mitchell H: The secretome of Campylobacter concisus. FEBS J 2010, 277:1606-1617.

23. Uzzau S, Fasano A: Cross-talk between enteric pathogens and the intestine. Cell Microbiol 2000, 2:83-89.

24. Fasano A, Fiorentini C, Donelli G, Uzzau S, Kaper JB, Margaretten K, Ding X, Guandalini S, Comstock L, Goldblum SE: Zonula occludens toxin modulates tight junctions through protein kinase $\mathrm{C}$-dependent actin reorganization, in vitro. J Clin Invest 1995, 96:710-720.

25. Hossain MM, Nakamoto H: Role for the cyanobacterial HtpG in protection from oxidative stress. Curr Microbiol 2003, 46:70-76.

26. Desai $B V$, Morrison DA: An unstable competence-induced protein, CoiA, promotes processing of donor DNA after uptake during genetic transformation in Streptococcus pneumoniae. J Bacteriol 2006, 188:5177-5186.

27. Abeyrathne PD, Daniels C, Poon KK, Matewish MJ, Lam JS: Functional characterization of WaaL, a ligase associated with linking O-antigen polysaccharide to the core of Pseudomonas aeruginosa lipopolysaccharide. J Bacteriol 2005, 187:3002-3012.

28. Yuan Y, Crane DD, Simpson RM, Zhu YQ, Hickey MJ, Sherman DR, Barry CE: The 16-kDa alpha-crystallin (Acr) protein of Mycobacterium tuberculosis is required for growth in macrophages. Proc Natl Acad Sci USA 1998, 95:9578-9583.

29. Wilkinson KA, Stewart GR, Newton SM, Vordermeier HM, Wain JR, Murphy HN, Horner K, Young DB, Wilkinson RJ: Infection biology of a novel alpha-crystallin of Mycobacterium tuberculosis: Acr2. J Immunol 2005, 174:4237-4243. 
30. Stewart GR, Newton SM, Wilkinson KA, Humphreys IR, Murphy HN, Robertson BD, Wilkinson RJ, Young DB: The stress-responsive chaperone alpha-crystallin 2 is required for pathogenesis of Mycobacterium tuberculosis. Mol Microbiol 2005, 55:1127-1137.

31. Stewart JN, Rivera HN, Karls R, Quinn FD, Roman J, Rivera-Marrero CA: Increased pathology in lungs of mice after infection with an alphacrystallin mutant of Mycobacterium tuberculosis: changes in cathepsin proteases and certain cytokines. Microbiology 2006, 152:233-244.

32. Ewing $C P$, Andreishcheva $E$, Guerry P: Functional characterization of flagellin glycosylation in Campylobacter jejuni 81-176. J Bacteriol 2009, 191:7086-7093.

33. Guerry P, Ewing CP, Schirm M, Lorenzo M, Kelly J, Pattarini D, Majam G, Thibault P, Logan S: Changes in flagellin glycosylation affect Campylobacter autoagglutination and virulence. Mol Microbiol 2006 60:299-311.

34. Logan SM: Flagellar glycosylation - a new component of the motility repertoire? Microbiology 2006, 152:1249-1262.

35. Schoenhofen IC, McNally DJ, Brisson JR, Logan SM: Elucidation of the CMPpseudaminic acid pathway in Helicobacter pylori: synthesis from UDP-Nacetylglucosamine by a single enzymatic reaction. Glycobiology 2006, $16: 8 C-14 \mathrm{C}$

36. Schoenhofen IC, Vinogradov E, Whitfield DM, Brisson JR, Logan SM: The CMP-legionaminic acid pathway in Campylobacter: biosynthesis involving novel GDP-linked precursors. Glycobiology 2009, 19:715-725.

37. Istivan TS, Coloe PJ, Fry BN, Ward P, Smith SC: Characterization of a haemolytic phospholipase $\mathrm{A}(2)$ activity in clinical isolates of Campylobacter concisus. J Med Microbiol 2004, 53:483-493.

38. Conesa A, Gotz S, Garcia-Gomez JM, Terol J, Talon M, Robles M: Blast2GO: a universal tool for annotation, visualization and analysis in functional genomics research. Bioinformatics 2005, 21:3674-3676.

39. Kanehisa M, Araki M, Goto S, Hattori M, Hirakawa M, Itoh M, Katayama T, Kawashima S, Okuda S, Tokimatsu T, Yamanishi Y: KEGG for linking genomes to life and the environment. Nucleic Acids Res 2008, 36: D480-484.

40. Stothard P, Wishart DS: Circular genome visualization and exploration using CGView. Bioinformatics 2005, 21:537-539.

41. Tanner ACR, Badger S, Lai C-H, Listgarten MA, Visconti RA, Socransky SS: Wolinella gen. nov., Wolinella succinogenes (Vibrio succinogens Wolin et al.) comb. nov., and description of Bacteroides gracilis sp. nov., Wolinella recta sp. nov., Campylobacter concisus sp nov., and Eikenella corrodens from humans with periodontal disease. Int I Syst Bacteriol 1981, 31:432-445.

doi:10.1186/1757-4749-3-15

Cite this article as: Kaakoush et al: Comparative analyses of Campylobacter concisus strains reveal the genome of the reference strain BAA-1457 is not representative of the species. Gut Pathogens 2011 3:15.

\section{Submit your next manuscript to BioMed Central and take full advantage of:}

- Convenient online submission

- Thorough peer review

- No space constraints or color figure charges

- Immediate publication on acceptance

- Inclusion in PubMed, CAS, Scopus and Google Scholar

- Research which is freely available for redistribution 